HAVANA, Cuba-As biotechnology | year. enters its second decade, the early expectation-that its products could help reduce the suffering caused by infectious diseases in developing countries-is nearly a reality. Two presentations at the international interferon and biotechnology meetings held here in April provide examples.

Rebecca and Ernesto Falcoff (Institut Marie Curie, Paris) and their collaborators, Roberto Badaro's group at the National University of Bahia (Salvador, Brazil), reported dramatic successes in treating antimonial resistant visceral leishmaniasis with $\gamma$-interferon. And Martin Lopez (Alexander von Humboldt University, Lima, Peru), along with his colleagues at the University of California (Berkeley) and the National Institute of Health (Lima), have developed an in vitro DNA amplification protocol to detect leishmania parasites in skin biopsies.

The leishmaniases comprise several diseases caused by different forms of the intracellular protozoa Leishmania. Worldwide, about 12 million people suffer from some form of leishmaniasis, with the most severe forms of the disease occuring in East Africa, Latin America, and India. Of the various forms, only visceral leishmaniasis is considered fatal if untreated. The last recorded epidemic, in India in 1978-79, caused 20,000 deaths. It is against this fatal form of the disease that $\boldsymbol{\gamma}$-interferon appears to show therapeutic efficacy.

In leishmaniasis (as well as several other diseases caused by intracellular parasites), the patient's $T$ cells are unable to respond to parasite-specific antigens and secrete immunomodulators such as interleukin-2 (IL-2) and $\gamma$-interferon $(\gamma$-IF $)$. The Falcoffs' group therefore treated leishmaniasis patients by intramuscular administration of $\gamma$-IF, hoping to bypass the parasite-induced $T$ cell refractivity and initiate a normal immune response. In the initial open trials, the researchers worked with eight adolescent patients whose disease was unresponsive to standard antimonial therapy, and nine patients with no previous treatment. All 17 were clinically cured after one or two cycles of approximately $50 \mu \mathrm{g}$ IF given daily for ten days. The first group has remained cured for one year, and the second has been followed now for six months. Based on these results, the scientists are organizing a larger placebo-controlled study to begin this
To underscore the generality and timeliness of this approach, a paper in the March 1989 issue of The Journal of Experimental Medicine (169:893-907) from Zanvil Cohn's laboratory at the Rockefeller University (New York, NY) in collaboration with Hansen's disease research centers in Addis Abba, Ethiopia and Carville, Louisiana, reports that subcutaneous administration of IL-2 successfully reconstructs an effective cell-mediated immune response in lepromatous leprosy patients.

To be maximally effective any disease control strategy must incorporate accurate early diagnosis of infection, and highly sensitive measures of parasite load following pharmaceutical intervention. For leishmaniasis such an assay now exists. Lopez and his collaborators have synthesized oli-

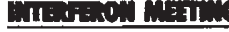

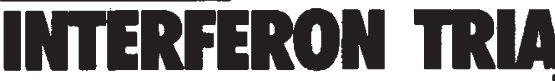

HAVANA, Cuba-Research scientists the world over-including from Yugoslavia, Italy, U.S.S.R., Sweden, China, and Argentina-continue to explore the possible clinical applications of the interferons, both natural and recombinant. And in Cuba, more than 10,000 patients over the last eight years have been treated with some form of interferon, according to Manuel Limonta, director of $\mathrm{Ha}-$ vana's Center for Genetic Engineering and Biotechnology. Speaking here in April at an international meeting that drew more than 1,200 participants from 50 countries, Limonta outlined some of these results. For viral diseases, he says, interferon seems to be effective against hemorrhagic dengue, hemorrhagic conjunctivitis, herpes and papilloma skin infections, hepatitis B (acute, chronic, carrier), and laryngeal papillomatosis. For neoplastic diseases-including chronic myeloid leukemia and low grade non-Hodgkin's lymphoma-treatment is most effective when the interferons are used together (alpha:gamma or natural alpha:recombinant alpha), or in combination with chemotherapy, radiotherapy, or surgery, says Limonta.

For acute hepatitis B infection (of which there are 50 million new cases worldwide every year), A. Sotto (Center for Biological Research, Havana) and his collaborators have treated 173 adult patients with alpha inter- gonucleotides that prime polymerase chain reaction (PCR) amplification of kinetoplast DNA sequences specific for Leishmania b. braziliensis. Using these primers, the scientists designed a hybridization assay for crude tissue homogenate that accurately identified the parasite in as little as $14 \mathrm{pg}$ of total DNA. It should be possible to identify such strain-specific amplification targets for the visceral leishmanias, and thus design a sensitive test to be used on splenic aspirants from patients in the interferon trials.

It is a bit ironic and a bit wonderful that now-when we know how to produce them so cheaply-the first glamour drugs of biotechnology are showing such promise in the treatment of what James Grant, the executive director of UNICEF, refers to as "diseases of the poor." Let's hope that this time we're right. - Harvey Bialy

\section{IS PROLIFERATE}

feron, using three different protocols. In the first, the patients received $15 \times 10^{6}$ units intraperitoneally over three days; the second group received the same dose over five days; and the third received $9 \times 10^{6}$ units over five days, intramuscularly. (There were 15 control patients.) The interferon treatment cured all the patients, and prevented the evolution of this disease to its chronic phase. Posttreatment liver biopsies showed that the tissue was not necrotic, and had even regenerated, says Sotto.

Chronic hepatitis B infection, on the other hand, is harder to treat with interferon. The 43 patients in this set of Cuban trials were randomized into two treatment groups: one received recombinant alpha-2 interferon for 14 weeks; the other was treated on the same schedule, but had previously been immunosuppressed with prednisone for two weeks. To determine if the treatment worked, the scientists measured patients' lymphocyte subpopulations with specific monoclonal antibodies. The group treated with prednisone showed an increased $\mathrm{T} 4$ cell population, and both groups had decreased T8 cell populations. Of the 18 patients who responded to this treatment, the majority ( 61 percent) had been immunosuppressed and had also been treated with leukocyte human interferon at least six months before being included in this study.-Jennifer Van Brunt 